\title{
A case of COVID-19 diarrhea relieved by bile acid sequestrant administration
}

\author{
Akira Shirohata $^{1} \cdot$ Ryusuke Ariyoshi $^{1} \cdot$ Seiji Fujigaki ${ }^{1} \cdot$ Katsuhide Tanaka $^{1} \cdot$ Teruhisa Morikawa $^{1} \cdot$ Tsuyoshi Sanuki $^{1}$. \\ Yoshikazu Kinoshita ${ }^{1}$
}

Received: 29 September 2021 / Accepted: 22 January 2022 / Published online: 4 February 2022

(c) Japanese Society of Gastroenterology 2022

\begin{abstract}
Patients with coronavirus disease 2019 exhibit various gastrointestinal symptoms. Although diarrhea is reported in many cases, the pathophysiology of diarrhea has not been fully clarified. Herein, we report a case of coronavirus disease 2019 with diarrhea that was successfully relieved by the administration of a bile acid sequestrant. The patient was a 59-year-old man whose pneumonia was treated by the administration of glucocorticoids and mechanical ventilation. However, beginning on the 30th hospital day, he developed severe watery diarrhea (up to 10 times a day). Colonoscopy detected ulcers in the terminal ileum and ascending colon. The oral administration of a bile acid sequestrant, colestimide, improved his diarrhea quickly. Ileal inflammation is reported to suppress expression of the gut epithelial apical sodium-dependent bile acid transporter. It decreases bile acid absorption at the distal ileum and increases colonic delivery of bile acids, resulting in bile acid diarrhea. In summary, the clinical course of the case presented in this report suggests that bile acid diarrhea is a possible mechanism of watery diarrhea observed in patients with coronavirus disease 2019 .
\end{abstract}

Keywords Bile acid sequestrant · Coronavirus disease $2019 \cdot$ Diarrhea $\cdot$ Gastrointestinal symptoms

\section{Introduction}

Since the end of 2019, the outbreak of coronavirus disease 2019 (COVID-19) has spread from China to the rest of the world, including Japan [1, 2]. Even now, COVID-19 is a serious international problem.

Patients with COVID-19 presents with a variety of symptoms including fever, dyspnea, sore throat, chills, and headache. They frequently complain of gastrointestinal (GI) symptoms in addition to respiratory ones [3]. GI symptoms may appear as the first manifestation in some cases [4]. Furthermore, patients with severe COVID-19 are at particularly high risk for developing GI complications [5].

GI symptoms includes anorexia, vomiting, nausea, diarrhea, GI bleeding, and abdominal pain. In particular, among GI symptoms, diarrhea has been reported in many cases, but the pathophysiology of the diarrhea in COVID-19 cases is

Akira Shirohata

whiteflag0229@gmail.com

1 Department of Gastroenterology, Steel Memorial Hirohata Hospital, 3-1 Yumesaki-cho, Hirohata-ku, Himeji, Hyogo 671-1122, Japan not completely understood. Schmulson et al. suggested that bile acid malabsorption (BAM) is an etiology of the diarrhea with COVID-19 [6]. There is, however, no case report to support this hypothesis. We report a case of COVID-19 with high-grade watery diarrhea that was successfully treated by the oral administration of a bile acid sequestrant. We suggested the possible role of bile acid diarrhea in the development of watery diarrhea in cases of COVID-19.

\section{Case report}

A 59-year-old man was admitted to Steel Memorial Hirohata Hospital for the treatment of high-grade watery diarrhea. He was treated at another hospital with mechanical ventilation and glucocorticoid administration for infection with severe acute respiratory distress syndrome coronavirus-2 (SARS$\mathrm{CoV}-2$ ) for over 4 weeks. During that hospitalization, his severe diarrhea exceeding $3 \mathrm{~L} /$ day developed on the 30 th day after the onset of COVID-19. The patient was treated with the administration of albumin tannate and probiotics with limited effect.

The patient was transferred to our department on the 40th day. His body weight was $97.6 \mathrm{~kg}$ and had increased 
in comparison with that before the onset of the disease. An abdominal contrast-enhanced computed tomography performed at that time showed severe intestinal edema, ascites and pleural fluid collection (Fig. 1). Although peripheral blood laboratory tests showed a significantly low serum albumin level of $1.3 \mathrm{~g} / \mathrm{dL}$, no other specific abnormalities were found, and the D-dimer level was already within normal range. The patient was treated with intravenous albumin administration and loop diuretics for 7 days. In addition, oral colestimide administration was started for diarrhea beginning on the 41 st day at a dose of $3.0 \mathrm{~g} /$ day (Fig. 2). A
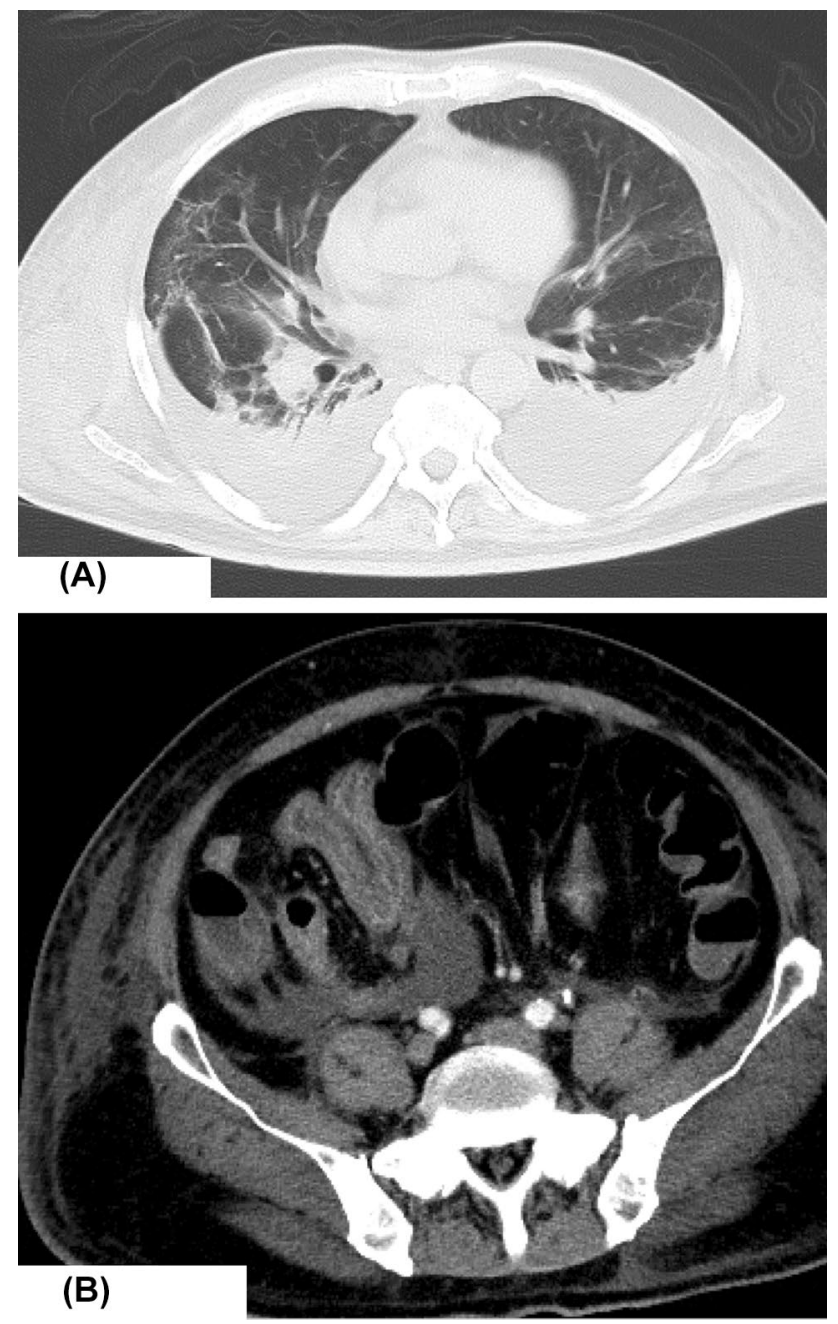

Fig. 1 Contrast-enhanced computed tomography images at the time of admission on the 40th day. A Severe bilateral pleural effusion and organized pneumonia. B Ascites in the abdominal cavity and swollen small intestine significant decrease in the amount of diarrhea was observed. The volume of watery diarrhea decreased to $460 \mathrm{~mL}$ on the 43rd day, which was less than half of the volume before colestimide administration. Its volume further decreased to $250 \mathrm{~mL}$ on the 47 th day. However, once the administration of colestimide was stopped on the day 47 , the volume of watery diarrhea increased to $600 \mathrm{~mL}$ the next day (Fig. 3). Moreover, stool culture submitted on admission showed no pathogenic bacteria suggestive for infectious enteritis, including Clostridium difficile.

In this patient, hematochezia appeared on the days 50 and 53; both instances required colonoscopic hemostasis. The colonoscopy at the time of the first hematochezia showed multiple simple ulcers from the ascending colon to the terminal ileum, and one of the ulcers in the ascending colon was accompanied by a visible vessel, which was considered to be the cause of the bleeding (Fig. 4). In addition, there were no disease-specific findings in the biopsy specimens obtained. Based on the results of the physical examination, blood laboratory tests, and endoscopic examination with biopsy, any cause of infectious enteritis could be ruled out (Table 1). We considered that the diarrhea to be caused by BAM due to COVID-19 infection, because it once improved with colestimide administration, and other diseases were excluded by this differentiation.

During treatment for the bleeding ulcers, his watery diarrhea worsened. Colestimide administration, which had been discontinued, was started again. With colestimide administration, diarrhea promptly improved. Colestimide administration was terminated after solid stools were observed on the 60th day. Subsequently, there was no recurrence of GI symptoms including diarrhea or GI bleeding. The patient continued rehabilitation and was discharged home on the 68th day.

\section{Discussion}

A case of diarrhea associated with COVID-19 was reported. Following treatment for the high-grade pneumonia, the patient recovered. Beginning on the 30th hospital day, he developed severe watery diarrhea, and he also was found to have ulcers in the terminal ileum and ascending colon. The administration of a bile acid sequestrant, colestimide, resolved the diarrhea quickly (Fig. 5).

SARS-CoV-2 is a type of coronavirus that attaches to and infects cells with angiotensin-converting enzyme 2 


\section{g/dL Trends in albumin during hospitalization}

3.5

3

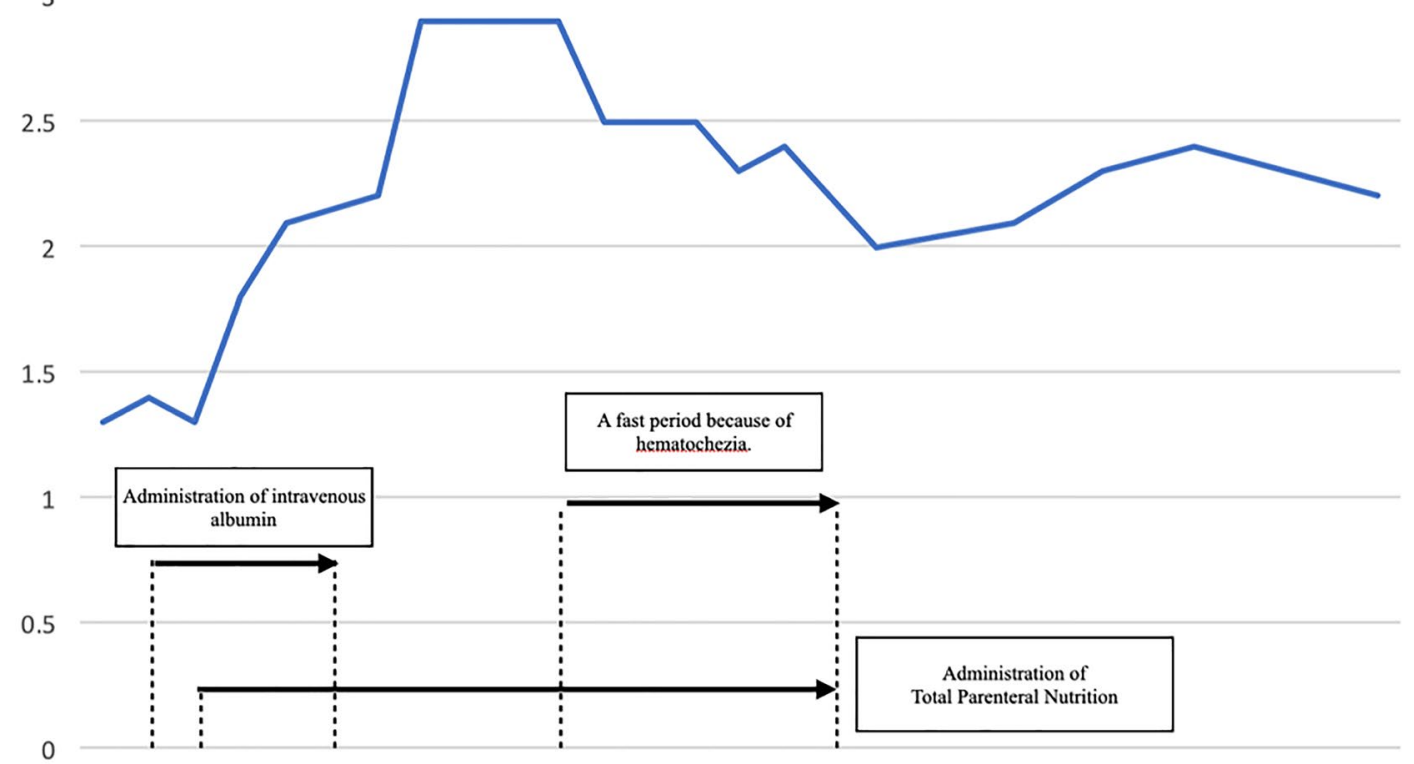

$\begin{array}{llllllllllllllllllllllllllllll}40 & 41 & 42 & 43 & 44 & 45 & 46 & 47 & 48 & 49 & 50 & 51 & 52 & 53 & 54 & 55 & 56 & 57 & 58 & 59 & 60 & 61 & 62 & 63 & 64 & 65 & 66 & 67 & 68 & \text { Day }\end{array}$

Fig. 2 Trends in albumin during hospitalization. Intravenous albumin was administered from the 41 st day to the 45 th day, and TPN was also administered from the 42nd day to the 56th day because the dietary intake was not stable. Thereby, on the 47 th day, the serum albu- min level improved to $2.9 \mathrm{~g} / \mathrm{dL}$. However, hypoalbuminemia occurred again on the 50th day caused by the development of hematochezia and a fast period of about 1 week. After restart of the diet, the hypoalbuminemia showed a gradual trend of improvement

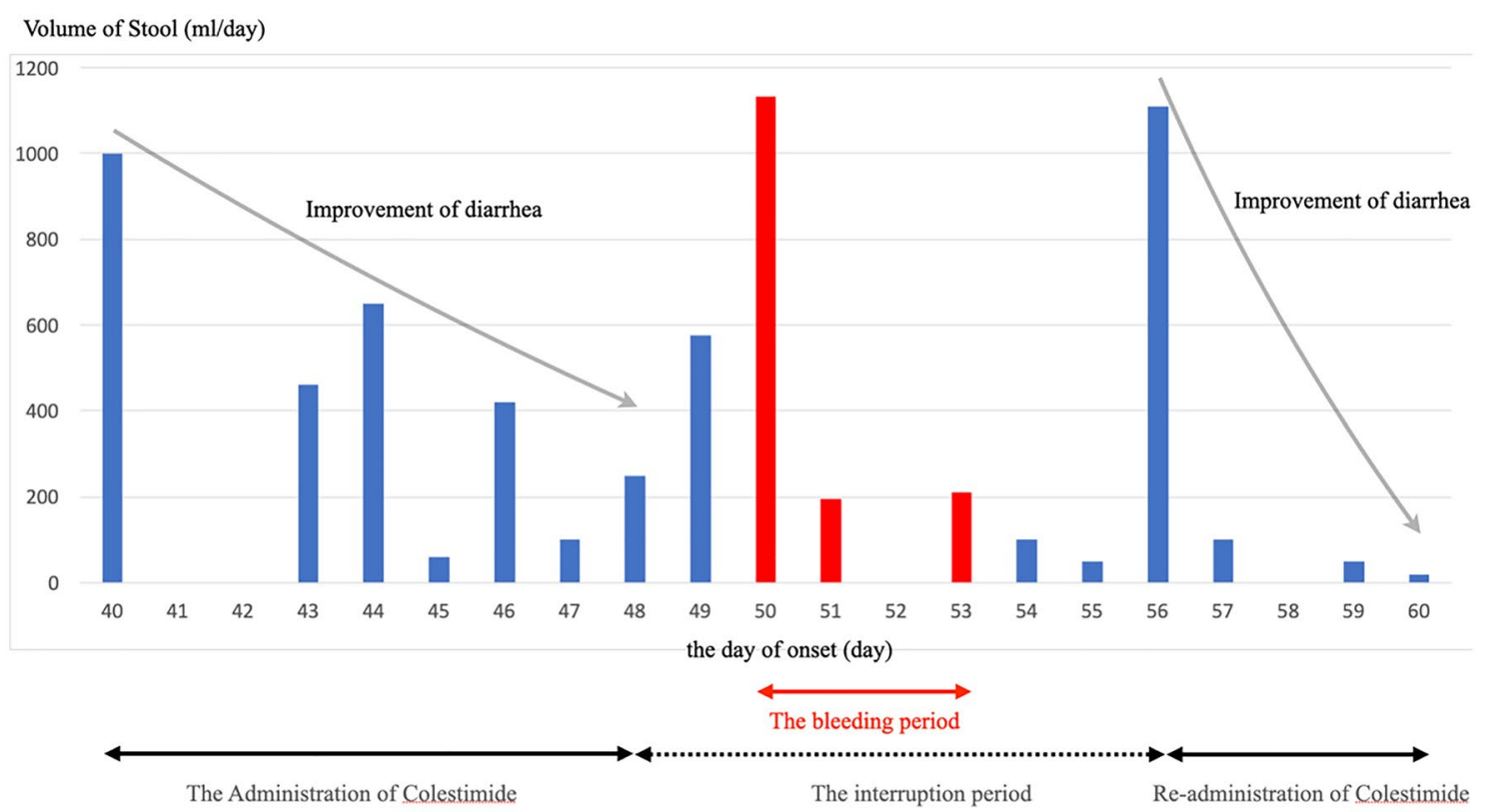

Fig. 3 Trends in stool volume after hospitalization. The volume of watery diarrhea decreased to $460 \mathrm{~mL}$ on the $43 \mathrm{rd}$ day, which was less than half of the volume before colestimide administration. Its vol- ume further decreased to $250 \mathrm{~mL}$ on the 47 th day. However, once the administration of colestimide was stopped on the day 47, the volume of watery diarrhea increased to $600 \mathrm{~mL}$ the next day 

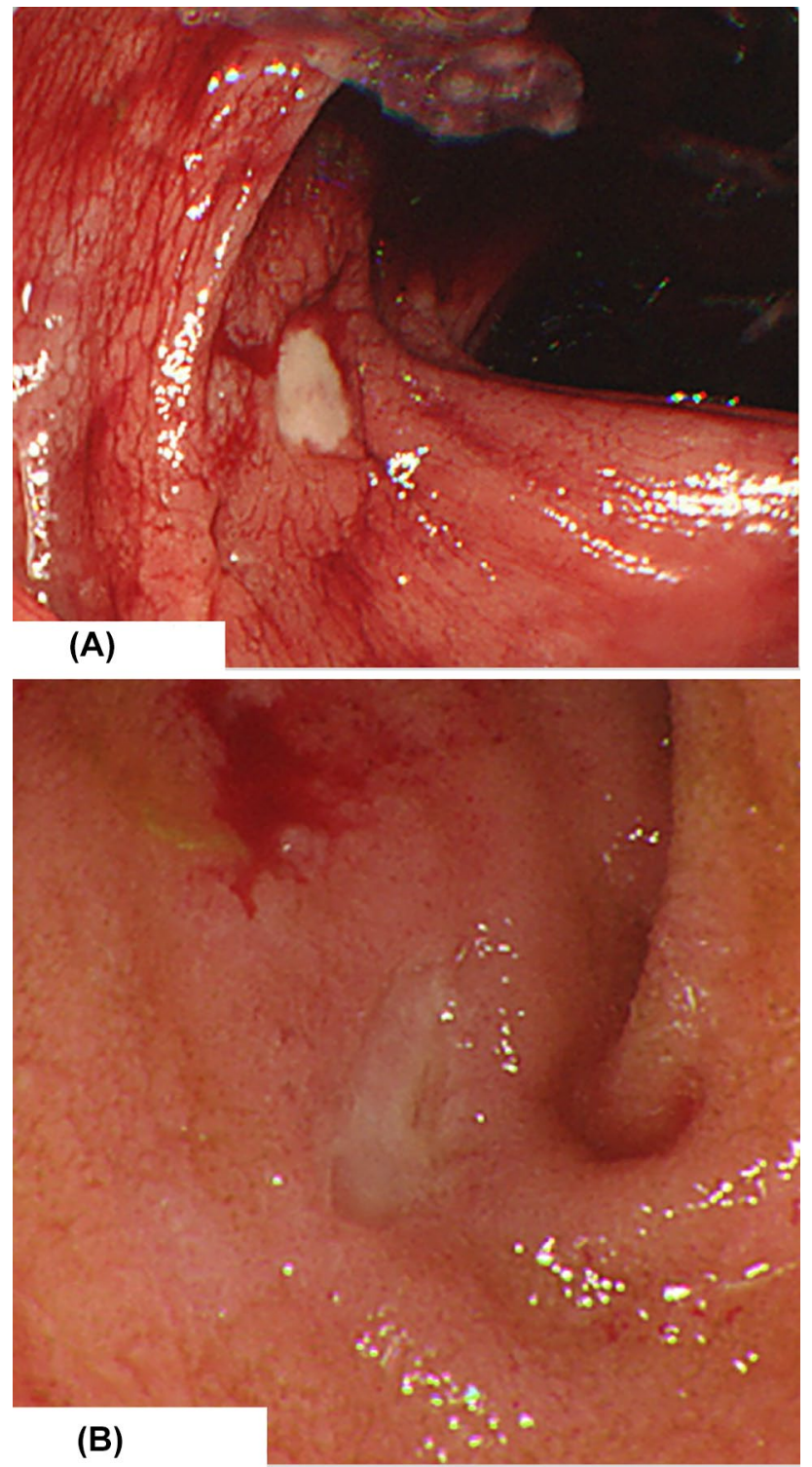

Fig. 4 Endoscopic image at the time of hematochezia on the 50th day. A Presented one of the ulcers in ascending colon. All ulcers, including this one, showed $5 \mathrm{~mm}$ in size and were small, round ulcers with smooth borders. B Presented one of the ulcers in terminal ileum. The biopsy results from this ulcer are loss of villi structure and inflammatory cell infiltration in the small intestine mucosa

(ACE2) receptors on their surfaces [6, 7]. ACE2 receptors are reported to be heavily expressed on the respiratory epithelial cells. In addition to the respiratory organs, the GI epithelial cells are reported to have ACE2 receptors and can be infected with SARS-CoV-2 [8]. Indeed, viral RNA are abundantly excreted in the feces of infected patients. Furthermore, subgenomic viral RNAs, which are found in the feces, show active viral replication in the GI mucosal cells [9]. Previous literature suggests that over $10 \%$ of patients with COVID-19 have GI symptoms, including diarrhea, and that GI mucosal inflammation may continue for a long period of time even after the pulmonary resolution of COVID-19 pneumonia [6, 9]. The COVID-19 patients with diarrhea are also reported to have elevated IL-6 in their plasma and increased calprotectin in their feces [10, 11].

On the other hand, the onset of diarrhea in this case was much later than the general onset date of diarrhea reported. [3]. As such, the diarrhea in this case was considered to be equivalent to post-COVID-19 syndrome (PCS). One of the pathogenic mechanisms of PCS is assumed to involve a persistent chronic inflammatory process [12]. The Rome Foundation Working team has reported the development of functional GI disorders (FGIDs) after infectious gastroenteritis or after resolution of an acute flare of inflammatory bowel disease $[6,13]$. These are referred to as post-infection or post-inflammatory FGIDs. Post-infection FGIDs (PI-FGIDs) have also been associated with dysbiosis and BAM. Notably, the resolution of SARS-CoV-2 infection resembles an aspect of PI-FGIDs, since patients with COVID-19 had significant alternations in their fecal microbiomes as compared to that in the controls. Moreover, it was reported that depleted symbionts and gut dysbiosis persisted even after clearance of SARS-CoV-2 and resolution of respiratory symptoms [9, 14]. Additionally, it has been reported that persistent dysbiosis leads to chronic intestinal inflammation [15]. Therefore, as with PI-FGIDs, it is natural to assume that the dysbiosis and BAM as a part of the chronic inflammatory process in PCS, similar to PI-FGIDs.

According to the clinical course of this case and the literature, we assumed a scenario for COVID-19-induced diarrhea (Fig. 6). At the time of the infection to the respiratory organs, diarrhea that occurs early in the development of COVID-19 is assumed to be due to infection of the small intestinal mucosa following the oral uptake of externalorigin SARS-CoV-2. Furthermore, regarding the duration of pneumonia, swallowing saliva that contains abundant SARS-CoV-2 in the airway mucus provides a continuous viral load to the small intestines. In addition, it was indicated that administration of PPI increases the $\mathrm{pH}$ of gastric acid, 
Table 1 Differential diseases in this case

\begin{tabular}{|c|c|}
\hline Differential diseases & Reasons for exclusion \\
\hline \multicolumn{2}{|l|}{ Infectious enteritis } \\
\hline Bacterial enteritis & Stool culture and endoscopic biopsy tissue culture were negative \\
\hline Cytomegalovirus enteritis & $\begin{array}{l}\text { Serologically, the CMV antigen C7-HRP was negative. No cytoplasmic inclusion bodies were found in tissue } \\
\text { samples by biopsy, and immunohistochemical staining using anti-CMV monoclonal antibody was negative } \\
\text { for CMV antibody }\end{array}$ \\
\hline Intestinal tuberculosis & $\begin{array}{l}\text { We did not measure tuberculosis-specific IFN- } \gamma \text { in the blood, but the patient had no history of pulmonary } \\
\text { tuberculosis, and there were no findings suggestive of obsolete pulmonary tuberculosis on chest CT scan. Fur- } \\
\text { thermore, there were no endoscopic findings such as ring ulcers or intestinal stenosis suggestive of intestinal } \\
\text { tuberculosis, and biopsy specimens did not point out any pyogenic granulomas }\end{array}$ \\
\hline \multicolumn{2}{|l|}{ Inflammatory bowel disease } \\
\hline Crohn's disease & $\begin{array}{l}\text { The patient had no history of IBD and no endoscopic findings, including cobblestone appearance and intestinal } \\
\text { stenosis, characteristic of Crohn's disease. In addition, there were no extraintestinal complications such as } \\
\text { anorectal lesions, skin lesions, or arthritis, and biopsy showed no granuloma species }\end{array}$ \\
\hline Behcet's disease & The patient did not have any oral aphthae, vulvar ulcers, or skin lesions during hospitalization or after discharge \\
\hline \multicolumn{2}{|l|}{ Drug-induced enteritis } \\
\hline $\begin{array}{l}\text { NSAIDs-induced small bowel } \\
\text { inflammation }\end{array}$ & The patient had not used any NSAIDs during hospitalization \\
\hline Collagenous colitis and sprue & $\begin{array}{l}\text { The patient was treated in the ICU on a ventilator for severe pneumonia caused by COVID-19. Of course, } \\
\text { esomeprazole was given to prevent stress gastric ulcer. However, disease-specific collagen bands could not be } \\
\text { indicated in the biopsy specimen in this case }\end{array}$ \\
\hline \multicolumn{2}{|l|}{ Vasculitis } \\
\hline $\begin{array}{l}\text { Lupus enteritis } \\
\text { ANCA-associated vasculitis }\end{array}$ & $\begin{array}{l}\text { ANCA and ANA measurements were not performed in this case. However, even though the serum markers for } \\
\text { each disease were positive, they were systemic diseases, and none of the symptoms or lesions could be shown } \\
\text { to satisfy the other diagnostic criteria }\end{array}$ \\
\hline \multicolumn{2}{|l|}{ The others } \\
\hline Amyloidosis & There was no amyloid deposition suggestive of amyloidosis \\
\hline Eosinophilic enteritis & There was and no eosinophilic infiltration suggestive of eosinophilic enteritis \\
\hline
\end{tabular}

possibly increasing the viability of the virus in the stomach, which can lead to COVID-19 exacerbations [16, 17]. For COVID-19 clearance, several reports indicated that SARSCoV-2 infection of the small intestine persists for 3-4 weeks after the first sputum specimen was PCR positive [3, 18]. Consequently, this prolonged and persistent infection of the small intestine leads to chronic intestinal inflammation due to dysbiosis and persistent diarrhea, which is similar to PI-FGIDs.

The presence of intestinal inflammation may cause diarrhea through some mechanisms, one of which is the decreased reabsorption of secreted bile acids at the terminal ileum. In healthy individuals, over $95 \%$ of the intestinal luminal bile acids are absorbed actively from the distal ileum and returned to the liver through the enterohepatic circulation [19]. The presence of ileal inflammation, especially the presence of the inflammatory cytokine IL-6, is reported to decrease the expression apical sodium-dependent bile acid transporter (ASBT), a pivotal bile acid absorption system on the apical epithelial surface of gut epithelial [10, 20-22]. Decreased ASBT expression is expected to decrease the bile acid absorption capacity at the distal ileum [23], which in turn, increases colonic delivery of bile acids. In the colon, the bile acids stimulates colonic water 

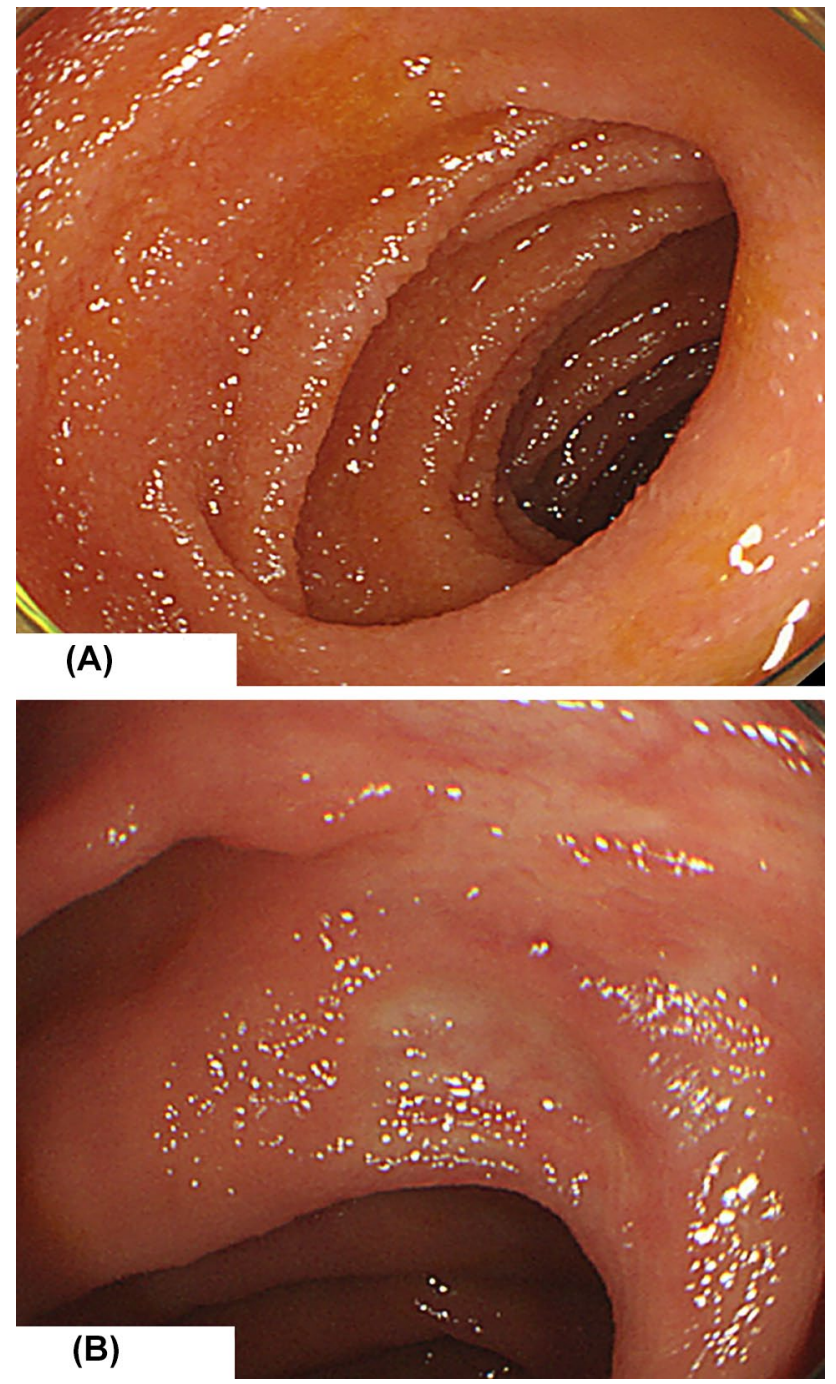

Fig. 5 Endoscopic image 3 months after discharge. There are no obvious abnormal findings on both images, and the ulcer and erosion had completely cured. A Presented a part of terminal ileum. B Presented a scar of the colonic ulcer

secretion and peristalsis, leading to bile acid diarrhea [19, 24].

The development of severe watery diarrhea and bleeding ileo-colonic ulcers of the patient in this report suggests the possible presence of chronic ileal inflammation even after the pulmonary resolution of the disease, and we associated PI-FGIDs and BAM with the persistence of diarrhea after resolution of the respiratory infection. Furthermore, it is already known that the administration of a bile acid sequestrant is effective in treating bile acid diarrhea and colestimide is clinically used for the treatment of bile acid diarrhea caused by a pathology in the terminal ileum, which is why it was used early in this case $[19,25,26]$. As a result, the administration of colestimide quickly improved the diarrhea quickly and diarrhea-related peripheral blood chemical abnormalities. Colestimide is an anion exchange resin that effectively binds bile acids in the gut. Therefore, if taken orally, it binds bile acids in the small intestinal and colon, which inhibits bile acid-induced colonic water secretion and peristalsis. The rapid effect of colestimide in this case suggests the role of bile acid in the development of watery diarrhea and the possible therapeutic role of bile acid sequestrants in patients with COVID-19.

Nevertheless, as a disease that presents with diarrhea and ulcers at the terminal ileum, there is a need to distinguish COVID-19-induced diarrhea from other diseases. Differential diagnoses of the inflammation of the small intestines including intestinal bowel disease, infection, drug-induced enteritis and vasculitis were all ruled out (Table 1). In addition, some studies have reported mucosal damage in the GI tract of patients with COVID-19 [27, 28]. This showed that SARS-CoV-2 infected the intestinal epithelium, thereby causing damage and inflammation. This inflammation could result in erosions and ulcers, subsequently reducing the expression of ASBT and causing bile acid diarrhea.

Finally, the diagnosis of bile acid diarrhea should ideally be based on the ${ }^{75}$ selenium homotaurocholic acid retention test $[19,29]$. However, this particular test is not available in Japan, making it the limitation of this case report. In many countries where this test is unavailable, the response to bile acid sequestrant is the internationally accepted method for the diagnosing of bile acid diarrhea [29, 30].

In summary, the clinical course of the case presented in this report suggests that bile acid diarrhea is a possible mechanism of chronic watery diarrhea observed in patients with COVID-19. 


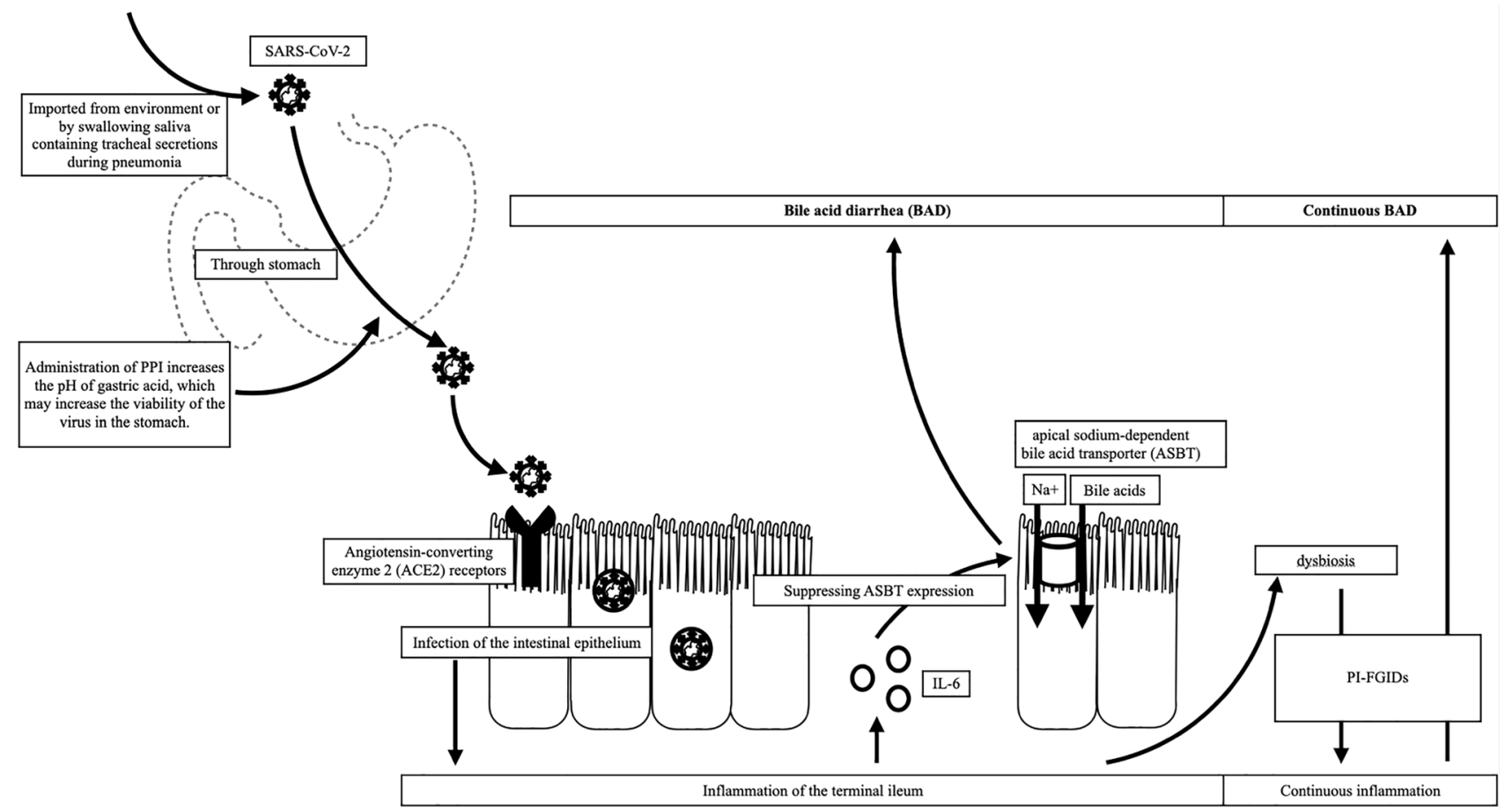

Fig. 6 Proposed a scenario of infection of severe acute respiratory distress syndrome coronavirus-2 (SARS-CoV-2), which leads bile acid diarrhea (BAD). At first, the diarrhea that occurs early in the development of COVID-19 is thought to be due to infection of the small intestinal mucosa by the virus through oral uptake of externalorigin SARS-CoV-2, at the time of the infection to respiratory organs. Then, for the duration of the pneumonia, swallowing saliva that contains abundant SARS-CoV-2 in the airway mucus provides a continuous supply of the virus to the small intestine. In addition, it was indicated that administration of PPI increases the $\mathrm{pH}$ of gastric acid, which may increase the viability of the virus in the stomach and lead to exacerbate COVID-19. Thus, SARS-CoV-2 frequently infects and

Acknowledgements The authors thank the staffs of the Endoscopy Center and the Section of Infection Control at the Steel Memorial Hirohata Hospital.

Author contributions AS and YK were involved in the case report design and data interpretation, the data analysis. All authors critically revised the report, commented on drafts of the manuscript, approved the final report, and agree to be accountable for all aspects of the work in ensuring that questions related to the accuracy or integrity of any part of the work are appropriately investigated and resolved.

\section{References}

1. Zhu N, Zhang D, Wang W, et al. A novel coronavirus from patients with pneumonia in China, 2019. N Engl J Med. 2020;382:727-33.

2. Watanabe M. The COVID-19 pandemic in Japan. Surg Today. 2020;50:787-93.

3. Tian Y, Rong L, Nian W, et al. Review article: gastrointestinal features in COVID-19 and the possibility of faecal transmission. Aliment Pharmacol Ther. 2020;51:843-51. proliferates in gut mucosal cells. The presence of ileal inflammation, especially the presence of an inflammatory cytokine IL-6, is reported to decrease the expression of gut epithelial apical sodium-dependent bile acid transporter (ASBT), a pivotal bile acid absorption system on the apical epithelial surface. Decreased ASBT expression is expected to decrease the bile acid absorption capacity at the distal ileum. The decreased absorption of bile acids at the distal ileum increases colonic delivery of bile acids and leads BAD. This prolonged persistent SARS-CoV-2 infection of the small intestinal mucosa leads to chronic intestinal inflammation by causing dysbiosis and continued diarrhea from various aspects which is similar to PI-FGIDs

4. Schmulson M, Dávalos MF, Berumen J. Beware: gastrointestinal symptoms can be a manifestation of COVID-19. Rev Gastroenterol Mex (Engl Ed). 2020;85:282-7.

5. Hayashi Y, Wagatsuma K, Nojima M, et al. The characteristics of gastrointestinal symptoms in patients with severe COVID19: a systematic review and meta-analysis. J Gastroenterol. 2021;56:409-20.

6. Schmulson M, Ghoshal UC, Barbara G. Managing the inevitable surge of post-COVID-19 functional gastrointestinal disorders. Am J Gastroenterol. 2021;116:4-7.

7. Xiao F, Tang M, Zheng X, et al. Evidence for gastrointestinal infection of SARS-CoV-2. Gastroenterology. 2020;158:1831-1833.e3.

8. Deshmukh V, Motwani R, Kumar A, et al. Histopathological observation in COVID-19. A systematic review. J Clin Pathol. 2021;74:76-83.

9. Zuo T, Liu Q, Zhang F, et al. Depicting SARS-CoV-2 faecal viral activity in association with gut microbiota composition in patients with COVID-19. Gut. 2021;70:276-84.

10. Ha S, Jin B, Clemmensen B, et al. Serotonin is elevated in COVID-19-associated diarrhoea. Gut. 2021;70:2015-7.

11. Effenberger M, Grabherr F, Mayr L, et al. Faecal calprotectin indicates intestinal inflammation in COVID-19. Gut. 2020;69:1543-4. 
12. Anaya JM, Rojas MA, Salinas ML et al (2021) Post-COVID syndrome a case series and comprehensive review. Autoimmun Rev 20:102947

13. Barbara G, Grover M, Bercik P, et al. Rome foundation working team report on post-infection irritable bowel syndrome. Gastroenterology. 2019;156:46-58.e7.

14. Zuo T, Zhang F, Lui GCY, et al. Alterations in gut microbiota of patients with COVID-19 during time of hospitalization. Gastroenterology. 2020;159:944-955.e8.

15. Barbara G, Feinle-Bisset C, Ghoshal UC et al (2016) The intestinal microenvironment and functional gastrointestinal disorders. Gastroenterology, 1305-18.

16. Price E. Could the severity of COVID-19 be increased by low gastric acidity? Crit Care. 2020;24:456.

17. Almario CV, Chey WD, Spiegel BMR. Increased risk of COVID19 among users of proton pump inhibitors. Am J Gastroenterol. 2020;115:1707-15.

18. Kato H, Shimizu H, Shibue Y, et al. Clinical course of 2019 novel coronavirus disease (COVID-19) in individuals present during the outbreak on the Diamond Princess cruise ship. J Infect Chemother. 2020;26:865-9.

19. Ticho AL, Malhotra P, Dudeja PK, et al. Intestinal absorption of bile acids in health and disease. Compr Physiol. 2019;10:21-56.

20. Neimark E, Chen F, Li X, et al. c-Fos is a critical mediator of inflammatory-mediated repression of the apical sodium-dependent bile acid transporter. Gastroenterology. 2006;131:554-67.

21. Lazaridis KN, Pham L, Tietz P, et al. Rat cholangiocytes absorb bile acids at their apical domain via the ileal sodium-dependent bile acid transporter. J Clin Invest. 1997;100:2714-21.

22. Craddock AL, Love MW, Daniel RW, et al. Expression and transport properties of the human ileal and renal sodium-dependent bile acid transporter. Am J Physiol. 1998;274:G157-69.
23. Oelkers P, Kirby LC, Heubi JE, et al. Primary bile acid malabsorption caused by mutations in the ileal sodium-dependent bile acid transporter gene (SLC10A2). J Clin Invest. 1997;99:1880-7.

24. Camilleri M. Bile acid diarrhea: prevalence, pathogenesis, and therapy. Gut Liver. 2015;9:332-9.

25. Niaz SK, Sandrasegaran K, Renny FH, et al. Postinfective diarrhoea and bile acid malabsorption. J R Coll Phys Lond. 1997;31:53-6.

26. Puleston J, Morgan H, Andreyev J. New treatment for bile salt malabsorption. Gut. 2005;54:441-2.

27. Vanella G, Capurso G, Burti C, et al. Gastrointestinal mucosal damage in patients with COVID-19 undergoing endoscopy: an international multicentre study. BMJ Open Gastroenterol. 2021;8:e000578.

28. Lin L, Jiang X, Zhang Z, et al. Gastrointestinal symptoms of 95 cases with SARS-CoV-2 infection. Gut. 2020;69:997-1001.

29. Sadowski DC, Camilleri M, Chey WD, et al. Canadian Association of Gastroenterology clinical practice guideline on the management of bile acid diarrhea. Clin Gastroenterol Hepatol. 2020;18:24-41.e1.

30. Wedlake L, A'Hern R, Russell D, et al. Systematic review: the prevalence of idiopathic bile acid malabsorption as diagnosed by SeHCAT scanning in patients with diarrhoea-predominant irritable bowel syndrome. Pharmacol Ther. 2009;30:707-17.

Publisher's Note Springer Nature remains neutral with regard to jurisdictional claims in published maps and institutional affiliations. 